\title{
Measurements of the Persistent Current Decay and Snapback Effect in Tevatron Dipole Magnets
}

\author{
G.V. Velev, P. Bauer, J. DiMarco, R. Hanft, M. Lamm, P. Schlabach, C. Sylvester, M. Tartaglia, \\ J.C. Tompkins
}

\begin{abstract}
A systematic study of the persistent current decay and snapback effect in the fields of Tevatron accelerator dipoles was performed at the Fermilab Magnet Test Facility (MTF). The decay and snapback were measured under a range of conditions including variations of the current ramp parameters and magnet operational history. The study has mostly focused on the dynamic behavior of the normal sextupole component. In addition, the paper presents the persistent current effects observed in the other allowed field harmonics as well. The results provide new information about the previously observed "excess" decay during the first several seconds of the sextupole decay during injection and the correlation between the snapback amplitude and its duration.
\end{abstract}

Index Terms - Magnetic Field Measurements, Superconducting accelerator magnets

\section{INTRODUCTION}

$\mathrm{I}^{\mathrm{N}}$ $\mathrm{N}$ the complex operation of modern superconducting accelerators, persistent current effects play a significant role due to their relatively large amplitudes and strong time dependencies [1]. They were first observed during Tevatron operation because of a large chromaticity variation during the dwell at injection and were referred to as dynamic effects. It was found that these variations were unacceptable for accelerator operation and corrections were developed [2].

To optimize these corrections for the ongoing Tevatron Run II, a detailed measurement program on the sextupole decay and snapback in the main dipoles was executed and results were reported elsewhere [3]-[4]. A new algorithm for Tevatron operation was proposed [5] and has been adopted recently [6].

Some of the persistent current changes in the magnetic field occur during a relatively short period of several seconds. A typical example is the fast field change, called snapback, which occurs in all allowed field multipoles, when the superconducting magnet is ramped up after a constant current plateau. It is particularly important to correct for this effect at

Manuscript received August 26, 2006. Work supported by the U.S Department of Energy.

All authors are with Fermilab, P.O. Box 500, Batavia, IL 60510, USA, (email: velev@fnal.gov; phone: 630-840-2203; fax: 630-840-8079).

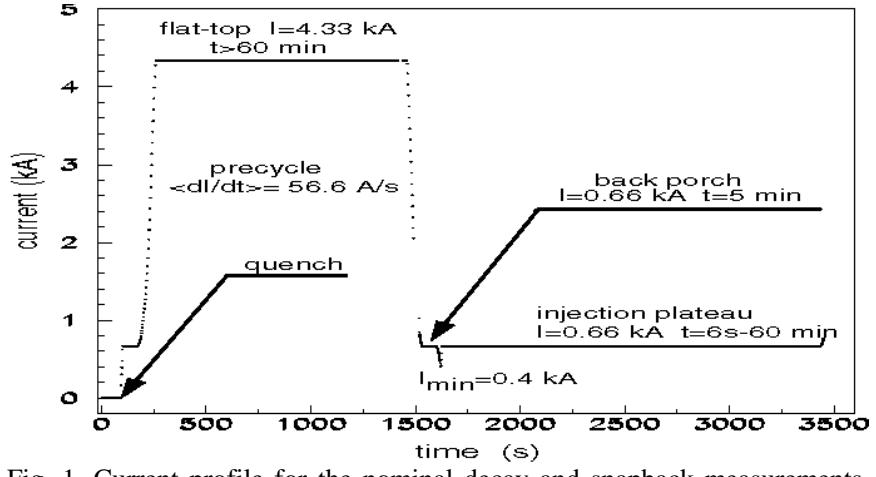

Fig. 1. Current profile for the nominal decay and snapback measurements. The only parameter varied is the duration of the injection plateau.

the beginning of an acceleration ramp.

The modified Tevatron operational ramp profile, adopted after our recent proposal for the optimization of the sextupole corrections during injection [6], is shown in Fig. 1. The minimal duration of the flattop is 60 minutes: used as a nominal value for our measurements. This time is needed to saturate the amount of field drift as a function of the previous magnet excitation, an effect known as "history dependence". The only parameter varied in our measurements is the duration of the injection plateau (IP): 6, 12, 20, 30, $60 \mathrm{~s}$ and 1, 5, 10, 15, 20, 25, 30 minutes are considered.

A new fast magnetic measurement system, based on the use of Digital Signal Processor (DSP) and Analog to Digital converter technology, was utilized. It gave us the ability to continuously sample the magnetic field using a rotating coil probe with a relatively high frequency, limited by the probe rotational speed (nominal value of $3 \mathrm{~Hz}$ is used for our measurements). More details about this system are reported elsewhere [7].

In this paper, we present a new study of the sextupole $\left(b_{3}\right)$ decay after very short injection plateau, summarize the results on the snapback and present new data on the dynamic effects in the main field and the higher order allowed harmonics in the Tevatron dipoles.

\section{SeXtupole Decay AND SNAPBACK}

\section{A. Sextupole Decay}

Sextupole decay in accelerator superconducting dipoles is a 


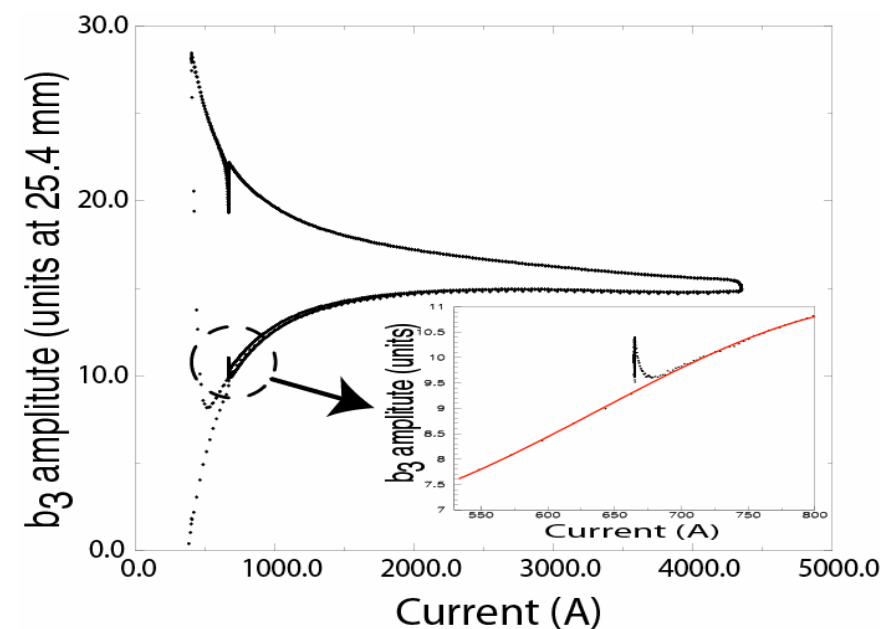

Fig. 2. Hysteresis loop of the sextupole field component. The inset shows the decay and snapback after $6 \mathrm{~s}$ of injection plateau and second order polynomial approximation under the region of interest.

well-known and more or less well-understood phenomenon. (See ref. [1]-[4] and the references found in [8].) However, recent Tevatron beam tests [6] and stand-alone magnet measurements [9] uncovered a new fast sextupole decay at the beginning of injection.

To quantify this fast decay, we analyzed the measurements on a set of spare Tevatron dipoles (TB0295, TB0491, TB0701, TB0834, TB1063, ТВ1065, ТС0710, TC0861, TC1047, TC1061, TC1077, TC1206) with the shortest available IP of $6 \mathrm{~s}$. A typical $\mathrm{b}_{3}$ hysteresis loop for TB1067 measurements with the decay and snapback is shown in Fig. 2. To decouple the decay from the underlying hysteresis loop, we parametrized $b_{3}$ as a function of current with a second order polynomial in the regions $0.56-0.65 \mathrm{kA}$ and $0.72-0.80$ $\mathrm{kA}$ and interpolated the $\mathrm{b}_{3}$ value at $0.66 \mathrm{kA}$ (Fig. 2 inset).

In the past, two different functional forms, logarithmic [2] and double exponential [10], have been used to model the long-term $b_{3}$ decay processes versus time in superconducting accelerator magnets. For the recent Tevatron correction algorithm, a modified logarithmic form

$$
\Delta b_{3}^{\text {dec }}=0.21+0.512 \cdot \log \left(\frac{t+170}{170}\right)
$$

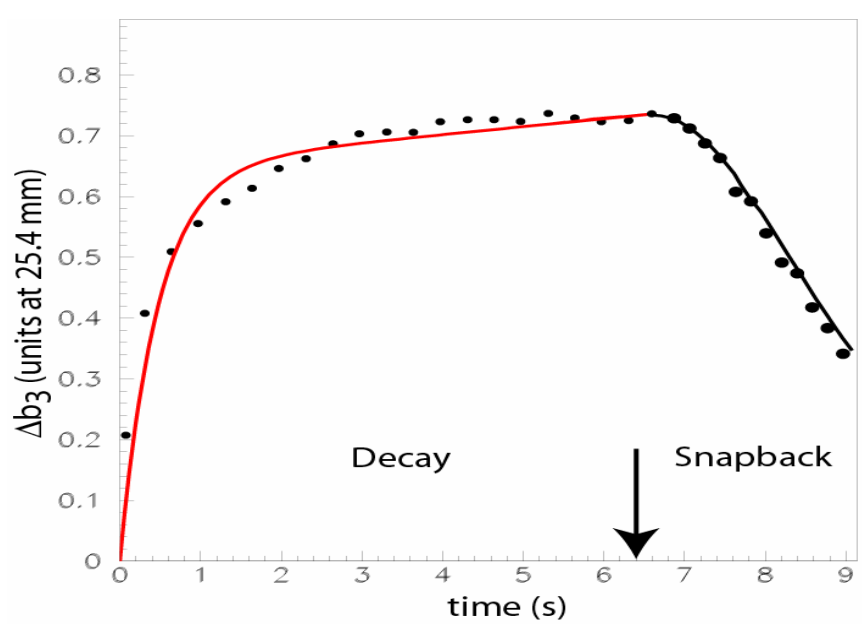

Fig. 3. $\quad \mathrm{A}_{3}$ field decay in TB1067 dipole after $6 \mathrm{~s}$ injection (left side of the picture). The decay line represents the fit with the functional form (2). Times after $6 \mathrm{~s}$ show the first few seconds of following snapback.
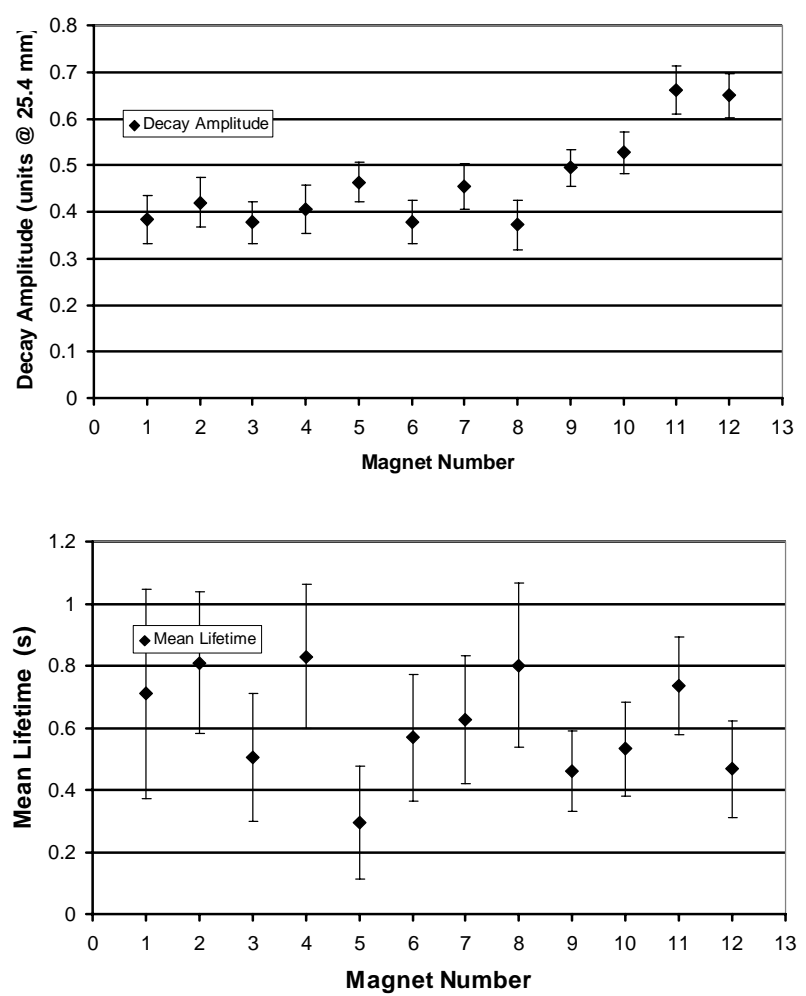

Fig. 4. The decay amplitudes, returned by the fit with functional form (2), for 12 Tevatron dipoles are shown on the top plot. The bottom plot shows the mean lifetimes. The magnet number corresponds to the order specified in the text.

is utilized (see ref. [6] for details). In (1), $t$ is the time on the injection plateau in seconds and the sextupole decay component $\Delta b_{3}{ }^{d e c}$ is in units.

Fig. 3 shows a $6 \mathrm{~s}$ decay for the TB1067 dipole. We found that a logarithmic function (1) could not accommodate for the fast decay in the first $2 \mathrm{~s}$ and decided to use the double exponential as proposed in [10]

$$
\Delta b_{3}^{\text {dec }}=b_{2,1} \cdot\left(1-\exp \left(\frac{-t}{t_{1}}\right)\right)+b_{2,2} \cdot\left(1-\exp \left(\frac{-t}{t_{2}}\right)\right) \text {. }
$$

The line in Fig. 3, in the region from zero to $6.4 \mathrm{~s}$, represents the fit with (2). The first exponent fits the short decay part while the second one, takes the long decay term. After $6.4 \mathrm{~s}$ the current ramp is activated and snapback starts.

A similar analysis was performed for eleven additional dipoles. The results are shown in Fig. 4. The upper plot summarizes the short-time decay amplitudes returned from the fit while the lower plot shows the measured mean lifetimes. (All errors represent one sigma standard deviation.) This short time decay has an average amplitude of $0.45 \pm 0.10$ units and mean lifetime of $0.61 \pm 0.06 \mathrm{~s}$. This is the first measurement of such fast decay in superconducting accelerator magnets.

\section{B. Snapback Measurements}

The stand-alone magnetic measurements have revealed two major features of the snapback: for the Tevatron case, the snapback is well described by a half Gaussian and its decay time $t_{\mathrm{sb}}\left(t_{\mathrm{sb}}=0\right.$ is at the beginning of the current ramp) is proportional to the magnitude of the drift. This variable $\left(t_{\mathrm{sb}}\right)$ 


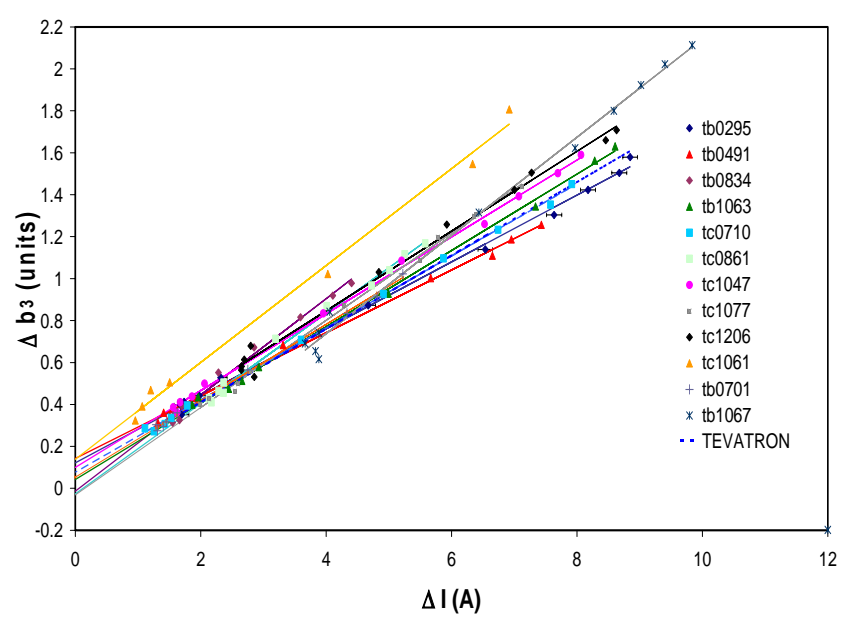

Fig. 5. The sextupole snapback amplitude $\Delta b_{3}{ }^{\text {sb }}(0)$ versus the snapback current for set of 12 Tevatron dipoles. The linear fits to the points are also shown, the dashed line represents the dependence on $\Delta I$, used in the Tevatron operation.

is directly connected to the current change $\Delta I$ at the beginning of the energy ramp. In the case of the Tevatron, the current profile at the beginning of the ramp has a parabolic dependence on time: $\Delta I=a t_{\mathrm{sb}}{ }^{2}$ where $a$ is the current ramp constant which is the same for the performed measurements. These results are summarized by following formulae

$$
\begin{aligned}
& \Delta b_{3}^{s b}(t)=\Delta b_{3}^{\text {dec }}\left(t_{\text {inj }}^{\text {end }}\right) \cdot \exp \left(\frac{-t^{2}}{t_{s b}^{2}}\right), \\
& \Delta b_{3}^{s b}(0)=\Delta b_{3}^{\text {dec }}\left(t_{\text {inj }}^{\text {end }}\right) \propto \Delta I .
\end{aligned}
$$

In the recently proposed scaling law [8], two main assumptions are made. The first assumption is that the proportionality, described in the second line of (3), is linear without an intercept term. The second assumption has a more general form and states that all the magnets of similar design (e.g. Tevatron dipoles) should follow the same linear dependence.

Fig. 5 shows the linear fits to the $\Delta b_{3}{ }^{\text {sb }}(0)$ dependence on $\Delta I$ for the examined dipoles. We found that the measured slope varies from magnet to magnet, in the range from 0.150 to 0.231 units/A with statistical and systematic uncertainty of 0.002 units/A. In addition, after combining the intercept values returned by the fits, we found $0.056 \pm 0.002$ units for the average intercept. These results do not agree with the scaling law statements of zero intercept and same magnet type linear dependence. Moreover, the observation of the non-zero intercept value is indirect confirmation of some fast non-linear process, occurring in the first several seconds of the decay at IP.

\section{DynAmic EFFECTS IN THE MAIN FieLD}

In a previous paper [9], we reported the results from the measurements of the decay in the main field for four Tevatron quadrupoles $\left(\mathrm{B}_{2}\right)$ after 30 minutes injection plateau. We found, in average, $0.67 \pm 0.10$ units change in $B_{2}$. The same analysis was performed on ten of the twelve measured Tevatron dipoles, utilizing our data sample with the longest injection plateau of 30 minutes. In two of the measured

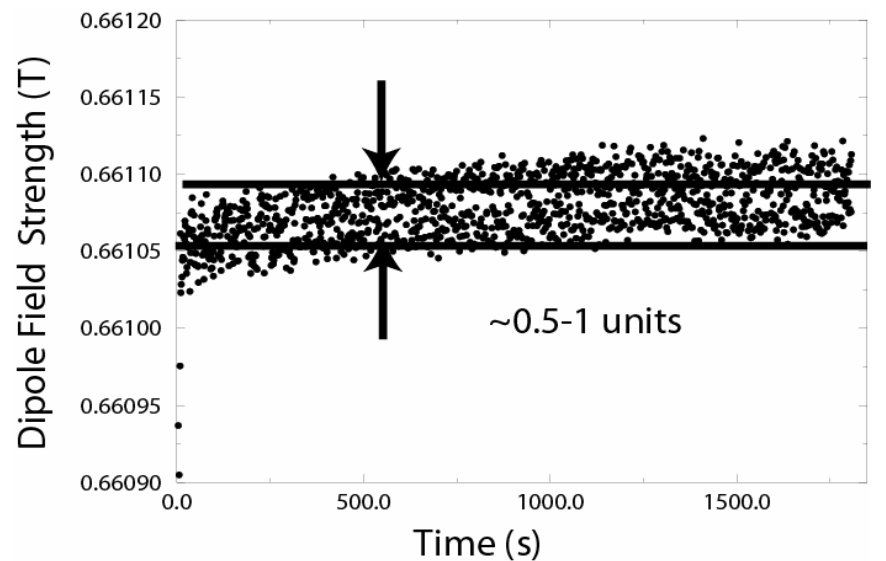

Fig. 6. Typical decay in the main dipole field after 30 minutes at injection plateau. A decay of 0.9 units is observed.

dipoles, we had a problem with current stabilization during IP. We did not include them in our analysis.

Typical main field decay in the dipole (TB0834) is shown in Fig. 6. The decay amplitudes of the other dipoles are in the range of 0.5 to 1.6 units with an average of 1.1 and a standard deviation of 0.3 units. Attempts to find the following snapback-type effect were unsuccessful. Taking into account the average decay change in the main dipole field of $7.35 \times 10^{-5} \mathrm{~T}$ and the current needed for this change, we estimated that the snapback should occur during a time interval of approximately $0.6 \mathrm{~s}$.

\section{Dynamic EFfect in Decapole ANd Tetradecapole}

We also investigated the dynamic effects in higher order allowed harmonics. The main idea is to compare the decay amplitudes and snapback times to the ones from the sextupole decay.

The amplitude of the decay processes decreases rapidly with the order of the multipoles. This effect can be observed in Fig. 6, which shows the decapole $\left(b_{5}\right)$ and tetradecapole $\left(b_{7}\right)$ decay compared to sextupole one in the magnet TB0701 after 30 minutes at IP. One can conclude that the level of the signal, coming from the dynamic effects, is limiting us to investigate the behavior of the higher than $b_{7}$ multipoles at this injection current.

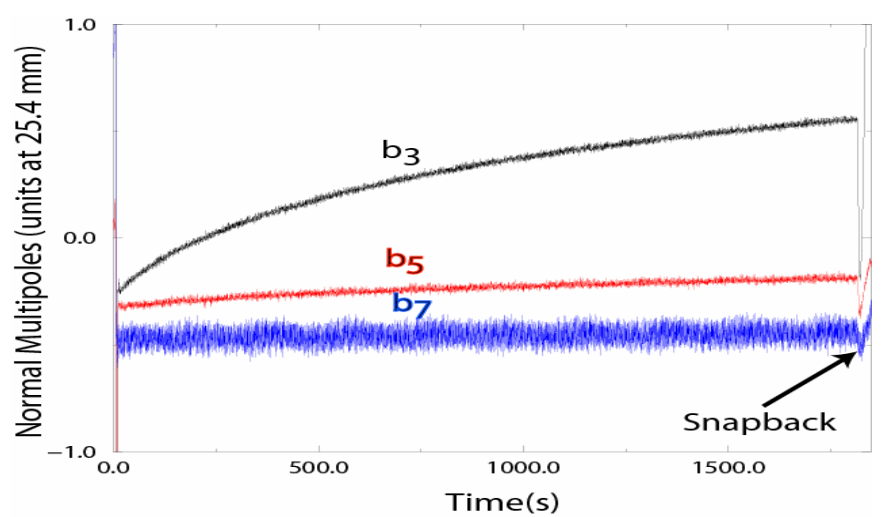

Fig. 7. Field decay in $b_{5}$ and $b_{7}$ multipoles (TB0701) after 30 minutes injection. For comparison, the sextupole $\left(b_{3}\right)$ decay is plotted also. The geometrical values of $b_{3}, b_{5}$ and $b_{7}$ are shifted arbitrarily for better presentation. 


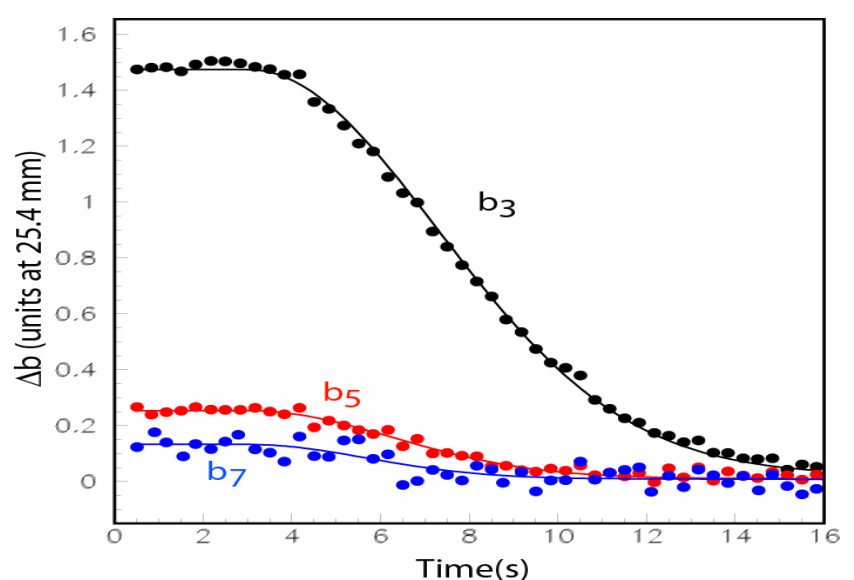

Fig. 8. The snapback for $b_{3}, b_{5}$ and $b_{7}$ field multipoles (TB0701) after 30 minutes injection. The curves are the Gaussian parameterization according to the first part of equation (3).

To characterize the $b_{5}$ and $b_{7}$ snapbacks, we performed a similar analysis as the one executed for $b_{3}$ [3], [4]. An example of the b5 and b7 parameterizations, according to the first part of (3), are shown in Fig. 8 and compared to the result from the $b_{3}$ analysis. One can observe that the ratio of the decay amplitudes is approximately $b_{3}: b_{5}: b_{7} \approx 10: 2: 1$.

Table I presents the results from the analysis of twelve Tevatron dipoles. The decay amplitude and snapback time in $b_{5}$ and $b_{7}$ multipoles are compared to the corresponding ones, measured from the $b_{3}$ dynamic effects. Assuming that the same linear scaling law (with the same proportionality constant for each magnet) found in sextupole is valid for $b_{5}$ and $b_{7}$, one should observe the following relation

$$
\Delta b_{k}^{s b} / \Delta b_{3}^{s b}=t_{s b, k}^{2} / t_{s b, 3}^{2}
$$

for $k=5,7$. The average values from Table I do not agree with (4) which points to different proportionality constants if the linear dependence $\Delta b_{k}^{s b} \propto \Delta I$ is preserved for $\mathrm{b}_{5}$ and $\mathrm{b}_{7}$ decays.

\section{CONCLUSION}

Using our fast DSP-based DAQ system [7], a detailed program of magnetic measurements was performed on a twelve Tevatron dipoles. For the first time, the relatively large decay found in Tevatron dipoles after very short injections, reported in [6] and [9], was measured. We determined its average amplitude and mean lifetime to be $0.45 \pm 0.10$ units and $0.61 \pm 0.06 \mathrm{~s}$ respectively.

In addition, an accurate check of the scaling law proposed in [8] was performed. For IP durations greater than $6 \mathrm{~s}$, we found the $\Delta b_{3}{ }^{s b}$ versus $\Delta I$ dependence is linear for the measured magnets. Moreover, the Tevatron dipoles show close but distinguishably different slopes, varying in the range 0.15-0.23 units/A, and non-zero intercept in the $\Delta b_{3}{ }^{s b}$ vs. $\Delta I$ evolution of the snapback.

For the first time we also report on the decay and snapback effects in the main field and allowed harmonics above $b_{3}$. For the main field in the Tevatron dipoles, we measured average
TABLE I

DeCAPOLE AND TETRADECAPOLE SNAPBACK PARAMETERS*

\begin{tabular}{ccccc}
\hline \hline $\begin{array}{c}\text { Dipole } \\
\text { name }\end{array}$ & $\begin{array}{c}\Delta \mathrm{b}_{5}{ }^{\mathrm{sb}} / \Delta \mathrm{b}_{3}{ }^{{ }^{\mathrm{ab}} \mathrm{b}} \\
\text { ratio }\end{array}$ & $\begin{array}{c}\Delta \mathrm{b}_{7}{ }^{\mathrm{sb}} / \Delta \mathrm{b}_{3}{ }^{\mathrm{sb}} \\
\text { amplitude } \\
\text { ratio }\end{array}$ & $\begin{array}{c}\mathrm{t}_{\mathrm{sb}, 5} / \mathrm{t}_{\mathrm{sb}, 3} \\
\text { snapback } \\
\text { time ratio }\end{array}$ & $\begin{array}{c}\mathrm{t}_{\mathrm{sb}, 7} / \mathrm{t}_{\mathrm{sb}, 3} \\
\text { snapback } \\
\text { time ratio }\end{array}$ \\
\hline TB0295 & 0.11 & 0.07 & 0.53 & 0.94 \\
TB0491 & 0.20 & 0.09 & 0.73 & 1.11 \\
TB0701 & 0.22 & 0.05 & 0.92 & 0.43 \\
TB0834 & 0.27 & 0.05 & 1.28 & 0.71 \\
TB1063 & 0.14 & 0.05 & 0.63 & 0.35 \\
TB1065 & 0.18 & 0.07 & 0.87 & 0.78 \\
TC0710 & 0.17 & 0.09 & 0.76 & 0.63 \\
TC0861 & 0.22 & 0.07 & 1.02 & 0.14 \\
TC1047 & 0.22 & 0.05 & 0.95 & 0.67 \\
TC1061 & 0.19 & 0.07 & 0.84 & 0.60 \\
TC1077 & 0.19 & 0.07 & 0.83 & 0.73 \\
TC1206 & 0.16 & 0.08 & 0.79 & 0.95 \\
Average & $0.19 \pm 0.04$ & $0.07 \pm 0.02$ & $0.85 \pm 0.19$ & $0.67 \pm 0.27$ \\
\hline \hline
\end{tabular}

* After a subtraction of the underlying hysteresis loop

decay amplitude of $1.1 \pm 0.3$ units after 30 minutes IP. Any attempt to find the following snapback-type effect was unsuccessful. We estimated that if the effect exists, it should occur for a period of approximately $0.6 \mathrm{~s}$.

The snapback amplitude and time in the $b_{5}$ and $b_{7}$ multipoles, after 30 minutes IP, are reported relative to the corresponding $\mathrm{b}_{3}$ parameters. We checked that sextupole linear dependence could not be applied directly to the $\Delta b^{\text {sb }}$ versus $\Delta I$ evolution of $b_{5}$ and $b_{7}$. Regardless of the fact that the $\Delta \mathrm{b}_{5}{ }^{\mathrm{sb}}$ decay amplitudes was found to be relatively small (e.g. $\Delta \mathrm{b}_{5}{ }^{\mathrm{sb}} / \Delta \mathrm{b}_{3}{ }^{\mathrm{sb}}=0.19 \pm 0.04$ ), the persistent current effects in the decapole may need to be taken into account in future LHC operation, where the injection plateau is expected to be relatively short and accurate dynamic corrections are critical to the initial beam stability.

\section{REFERENCES}

[1] D. A. Finley et al., "Time dependent chromaticity changes in the Tevatron”, in Proc. 1987 PAC., Washington, DC, 1987, pp. 151-153.

[2] D. E. Johnson and D.A. Herrup "Compensation of time varying field in the Tevatron superconducting magnets", in Proc. 1989 PAC., Chicago, 1989, pp. 521-523.

[3] G. V. Velev et al., "Measurements of field decay and snapback effect on Tevatron dipole magnets”, in Proc. 2003 PAC, Portland, 2003, pp. 19741976.

[4] G. V. Velev et al., "Measurements of sextupole decay and snapback in Tevatron dipole magnets" in Proc. $9^{\text {th }}$ EPAC, Lucerne, 2004, pp. 17801782.

[5] P. Bauer et al., "Proposals for improvements of the correction of sextupole dynamic effects in Tevatron dipole magnets" in Proc. $9^{\text {th }}$ EPAC, Lucerne, 2004, pp. 818-820.

[6] M. A. Martens et al., "Studies of the chromaticity, tune and coupling drift in the Tevatron", in Proc. 2005 PAC, Knoxville, 2005, pp. 725-727.

[7] G.V. Velev et al., "A fast continuous magnetic field measurement system based on digital signal processors", IEEE Trans. Appl. Supercond., Vol. 16, No. 2, 2006, pp. 1374-1377.

[8] G. Ambrosio et al., "A scaling law for predicting snapback in superconducting accelerator magnets”, IEEE Trans. Appl. Supercond., Vol. 15, No. 2, 2005, pp. 1217-1220.

[9] G. V. Velev et al., "Measurements of field decay and snapback effect on Tevatron dipole and quadrupole magnets", in Proc. 2005 PAC, Knoxville, 2005, pp. 2098-2100. 
[10] W. Fisher, A. Jain and S.Tepikian, "Beam-based measurements of the persistent current decay in RHIC”, in Proc. 2001 PAC, Chicago, 2001, pp. 2854-2856. 